\title{
Generation Algorithm of Radar Simulation Data Based on Electronic Chart Data
}

\author{
Hua Chen ${ }^{12}$ \\ Key Laboratory of Maritime Dynamic Simulation and Control of Ministry of Transportation, Dalian \\ Maritime University, Dalian 116026, China \\ E-mail: ch07790163.com
}

\section{Xinyu Zhang ${ }^{3}$}

Key Laboratory of Maritime Dynamic Simulation and Control of Ministry of Transportation, Dalian Maritime University, Dalian 116026, China

Faculty of Infrastructure Engineering Dalian University of Technology, Dalian 116024, China

E-mail: zhangxyedlmu.edu.cn

\section{Shun Yao, Changbin $\mathbf{X u}$}

Key Laboratory of Maritime Dynamic Simulation and Control of Ministry of Transportation, Dalian Maritime University, Dalian 116026, China

E-mail:ysdeemaile163.com, 180426804710163.com

\begin{abstract}
In order to generate a continuous radar simulation image to meet the practical need of navigation simulator, a method is presented to generate radar simulation data extracted from multi-scale S-57 standard electronic charts. In this paper, outliers index algorithm based on forward distance is presented firstly to clip the boundary line. Then buffers are built to merge and de-duplicate data according to characteristics of the data fragmentation. Finally, the average edge-matching method and the attenuation edge-matching method based on error distribution theory are provided to connect the gap automatically between two images according to the scale and the data density of charts. In this paper, 13 pieces of S-57 standard electronic charts around Rizhao Port are taken as an example. The simulation results show that the radar image is clear and the line is smooth, which can reach the purpose of physical and logical seamless contiguity of the radar image. In conclusion, the generated radar simulation data meet the requirements for continuity of the radar simulation image in the navigation simulator.
\end{abstract}

CENet2015

12-13 September 2015

Shanghai, China

\footnotetext{
${ }^{1}$ Speaker

${ }^{2}$ This research was financially supported by National Natural Science Foundation of China (Grant No.: 51309043), Applied Basic Research of Ministry of Transport (Grant No.: 2014329225020), Fundamental Research Funds for the Central Universities (Grant No.: 3132014202), China Postdoctoral Science Foundation (Grant No.: 2014M551095), Outstanding Young Scholars Growth Plan of Liaoning Province (Grant No.: LJQ201405) and Liaoning Provincial Natural Science Foundation of China (Grant No.: 2014025005).

${ }^{3}$ Correspongding Author
} 


\section{Introduction}

Currently, the navigation simulator (certified by Det Norske Veritas, DNV A-class) researched and developed independently by Dalian Maritime University, generates radar image based on the radar simulation data. The radar simulation data are extracted from S-57 standard electronic charts. It takes the vessel's position as the center, uses the method of circular scanning to find the intersection of each instant scan lines and target segments, and then extends the direction from the intersection to the target inside along the scanning line. Finally, it uses the method of echo generation based on Bresenham algorithm to generate the real time radar image [1]; however, there are some limitations in terms of using this method to generate the radar image. It can only read one piece of S-57 standard electronic chart data, when the simulated vessel steers beyond the scope of a single chart, the image will be inaccurate or even disappeared. Thus it is necessary to use more than one S - 57 standard electronic chart merging into a wide range of radar image. In order to solve this problem, Yao shun has presented an algorithm based on the forward angle to cut off the boundary line and used a unilateral attenuation method to connect the gap between two charts [1]. The algorithm can realize seamless contiguity of radar images, but it will accidentally delete radar data in cutting off boundary line and merging radar data, which will reduce the accuracy of image generation.

In the field of cartography and geographic information system, there are many researches on data processing and image stitching. Sui Chunguang gave specific steps to realize the seamless contiguity of digital chart [2]. Han Guangshun analyzed the relationship between map and feature, he uses the key technology and software to realize physical seamless contiguity of crossing feature [3]. Liuyun presented the idea of large scale topographic maps data splicing program and finally finishes working by Man-machine interactive ways [4]. Huang Huiping put forward the restrictive conditions and algorithmic of intelligentized edge method about intersected contour lines and uses ArcGIS Engine to realize the method [5]. Zhang Ying presented a virtual stitching algorithm based on vector ID mapping to improve the efficiency of automatic maps edge matching of massive digital map data in great regions even global ones [6]. Pouderoux J and Sandhya B researched on splicing for contour map with the same scale in GIS [7-8]. The above methods have made some achievements in teams of seamless contiguity of data, but are limited to the same scale of map sheet stitching.

In order to process radar data from different kinds of scales and generate more accurate radar simulation data, in this paper, the characteristics of radar data are fully exploited and the generation algorithm of radar simulation data based on electronic chart data is presented. As seen from the simulation results, the generated image is accurate and the line is smooth, which can basically maintain the original trend of lines.

\section{Generation Algorithm}

Three principles are included in the generation algorithm of radar simulation data based on electronic chart data:

Boundary line clipping, the radar data are preprocessed firstly and then outliers index algorithm based on forward distance is presented to cut off the boundary line.

Radar data merging and buffers are built to merge and de-duplicate data according to the characteristics of the data fragmentation.

The edge-matching, the average edge-matching method and the attenuation edge-matching method based on error distribution theory are provided to connect the gap between two images automatically. 


\subsection{Clipping}

Firstly, the original data are preprocessed to extract radar data to meet the needs of radar image generation. As seen in Fig. 1: the radar data form a closed polygon, in order to clip the boundary line which is not needed, then outliers index algorithm based on forward distance is presented.

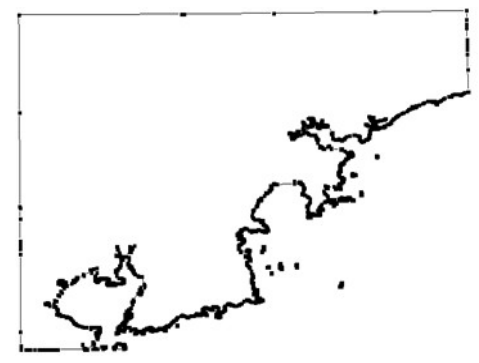

Figure 1: Original Chart C1312300 with A Boundary Line

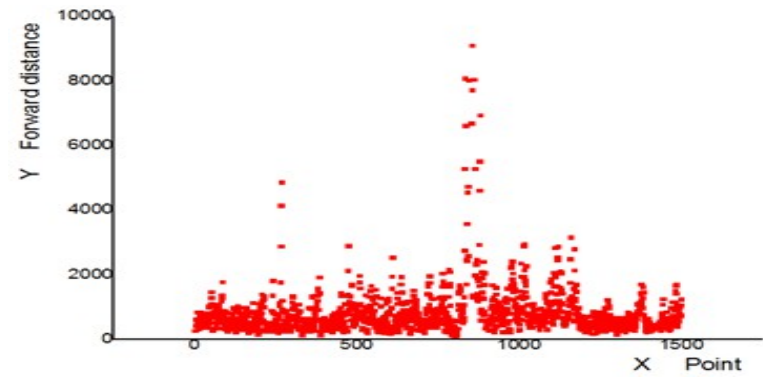

Figure 2: Scatter Diagram of the Forward Distance

\subsubsection{Data preprocessing}

The original data extracted from S-57 standard electronic chart include geographic position coordinates, attribute information and beacon, etc. In order to extract the radar data and convert them into the requiring format, the program of data extraction and conversion is needed.

\subsubsection{Outliers Index Algorithm based on Forward Distance}

From Fig. 1, we can find it easily that points which composite the boundary line are decentralized with respect to that of the coastline; thus the clipping algorithm is put forward based on the following steps:

Search each point's neighbor in the range of forward radius R;

Suppose $\mathrm{M}$ be the maximum number of an object point in the range of forward radius $\mathrm{R}$;

If the number $\mathrm{M}+1$ is found, the object point won't be an outlier; otherwise, it will be an outlier;

If the object point is an outlier, it will be deleted; otherwise, it will be kept.

Users can set their own forward radius and maximum number $M$ according to the characteristics of different charts. The setting method of $\mathrm{R}$ based on the following:

The formula $\sqrt{\left(x_{1}-x_{2}\right)^{2}+\left(y_{1}-y_{2}\right)^{2}}$ is used to find the distances between each point and its neighbor points. Users can set their own number of neighbor points by observing the original closed graph. For example, by observing Fig. 1, the maximum number $\mathrm{M}$ is set as 5 and the average distance between each point and its forward 5 points is found. As seen from Fig. 2, we can find that the distances more than 3000 are decentralized while the distances less than 3000 are dense. So we set $\mathrm{M}$ as 5 and $\mathrm{R}$ as 3000 to clip the boundary line of Fig. 1, the result can be seen in figure 3. From Fig. 3 we can get the idea that the boundary line has been cut off and the coastline remains the same so that the purpose of this clipping algorithm can be achieved.

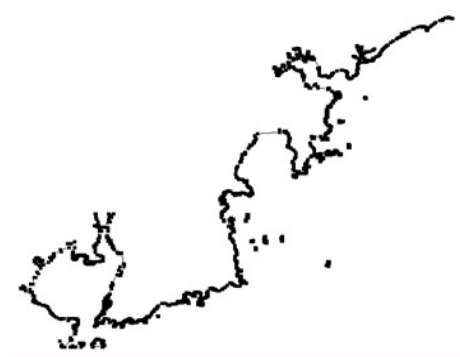

Figure3: Chart C1312300 with Boundary Line Clipped

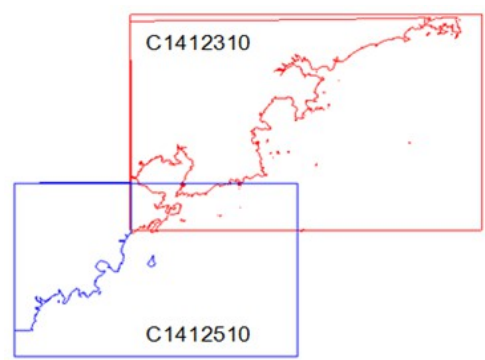

Figure 4: Coastline Divided by Different Charts 


\subsection{Merging}

Vessels sailing at sea usually use nautical charts for route planning. In order to design a more efficient and more accurate route between different charts, in the production of nautical charts retain a certain overlapping part between the adjacent charts, which will result in duplicated information acquisition and storage and it's a waste of resources [9]. In order to delete redundant data, an appropriate algorithm is needed to delete the low precision data and reserve the high precision data.

For the same object, sophistication and complexity in a large scale map are smaller than that in a small scale map [10]; thus we can believe that large scale chart data are more accurate than small scale chart data. If there is an overlapping portion in two charts with the same scale, the accuracy of high density chart data is obviously higher than the one with low density. Before merging, a data list is built with the data accuracy in a descending order, that is to say, the header of the data list with the highest accuracy while the last with the lowest accuracy.

The merging algorithm is shown in Fig. 5. Firstly, enter the header chart data of the data list, then enter the next chart data after the header, judge whether they have the same scale. If they are, analyze their density and take the chart data with high density as a benchmark; if the scale is not equal, take the chart data with large scale as a benchmark. Then the benchmark chart data are divided into several segments according to their characteristics. For each segment data, a buffer is built. If the low accuracy data fall into any one of the buffers, delete it; otherwise, reserve it. Finally, use the merged data as a new chart data and continue to merge with next chart data until all of the chart data have been merged.

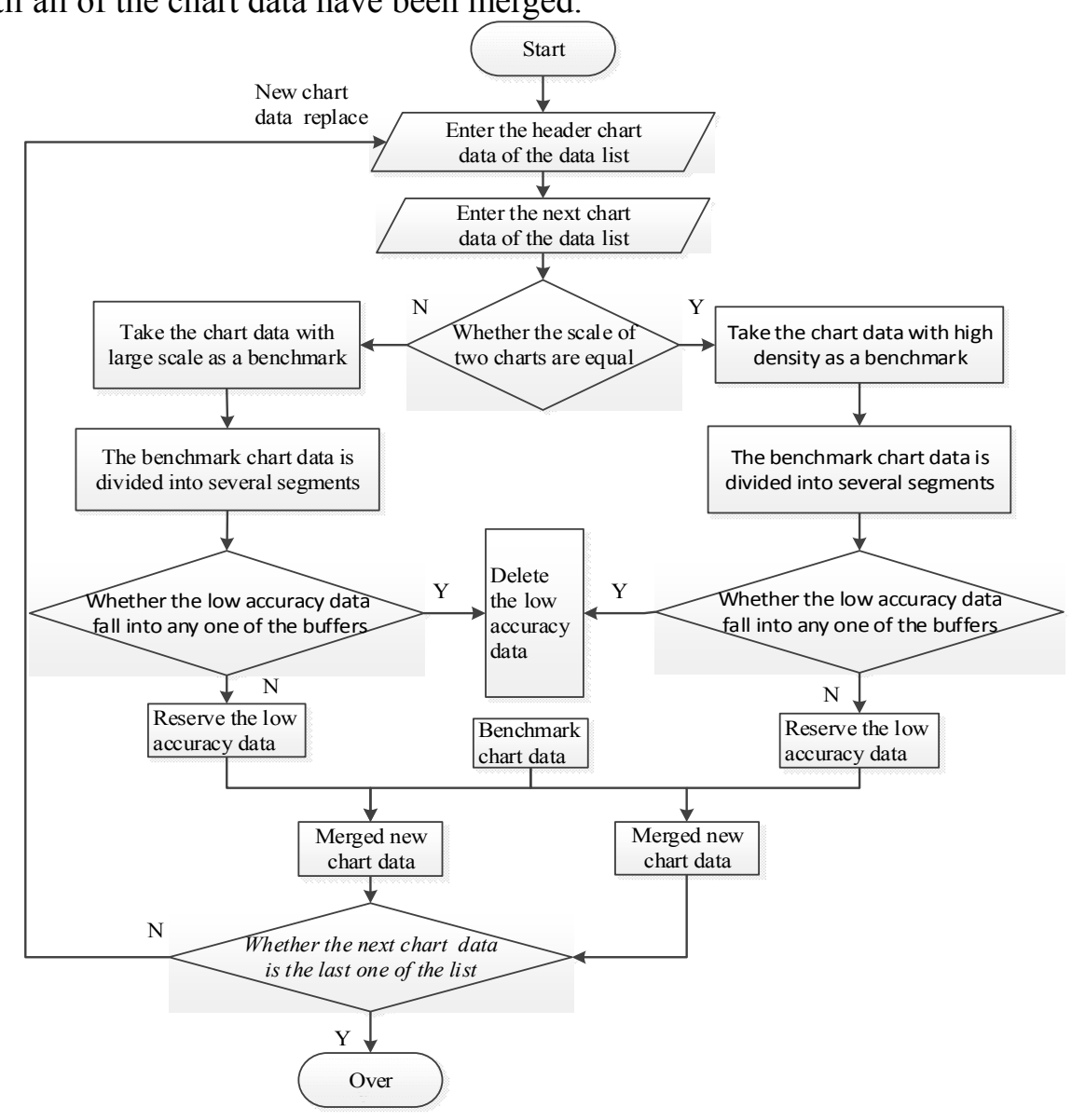

Figure 5: Merging Algorithm

\subsection{Edge-matching}

The existence of the gap between two images makes originally continuous coastline interrupted, which will cause a blank area when used in the marine simulator. In order to fill the 
blank area and make the image maintaining the original trend, this paper describes the reasons for causing the blank area firstly, then the edge-matching idea is given; finally, the average-edge matching method and the attenuation edge-matching method based on error distribution theory are put forward.

\subsubsection{Reasons for Causing the Blank Area}

In the process of S-57 standard electronic chart production, a complete feature will inevitably be separated into two or more data files, which make the same object divided into several parts. As can be seen in Fig. 4, a continuous coastline is divided into different charts.

\subsubsection{Idea of Edge-matching}

Before stitching coastline, the elements to be matched must be determined first because the chart data are stored in a file in different segments upon merged; thus it provides that the starting point of a line can only be connected to the last point of another line. In this paper, all first points and all last points of all segments are found and stored in two arrays respectively; besides, as to the first points in the first-point array and the last points in the last-point array, each first point searches for its nearest last point and each last point searches for its nearest first point. If the two distances are equal, different connection methods are taken according to the original chart scales belong to the two points. Currently, all electronic chart data are twodimension data. As a result, if the two original chart scale are equal, the average edge-matching method is taken; otherwise, the attenuation edge-matching method based on error distribution theory is taken.

\subsubsection{Average-edge Matching Method}

If the gaps between two charts in the same scale, the average edge-matching method is given, that is, to take the first point of one line and the last point of the other and use their average value as a midpoint. Suppose the two points to be connected as $\left(x_{1}, y_{1}\right)$ and $\left(x_{2}, y_{2}\right)$, the midpoint coordinates shall satisfy the following formula:

$$
\begin{aligned}
& x_{3}=\left(x_{1}+x_{2}\right) / 2 \\
& y_{3}=\left(y_{1}+y_{2}\right) / 2
\end{aligned}
$$

\subsubsection{Attenuation Edge-matching Method Based on Error Distribution Theory}

If the gaps between two charts in different scales, the attenuation edge-matching method based on error distribution theory is put forward, that is to take the large-scale data as standard, for small scale chart data, the error is allocated to the point to be connected and its adjacent points. According to the errors distribution theory in surveying, each point is regarded as a station, using the on-line distance as the weight to distribute error [11].

Assume the point coordinate be $\left(x_{1}, y_{1}\right)$ before matching and the point coordinate be $\left(x_{2}, y_{2}\right)$ after matching. The edge-matching error is $\Delta x=x_{1}-x_{2}$ and $\Delta y=y_{1}-y_{2}$, the error is distributed to a number of adjacent points. $P_{n}$ is the distance between the point to be connected and its adjacent points. After connection, we can get the point coordinate using the following formula:

$$
\begin{aligned}
& x_{n}^{\prime}=x_{n}+p_{n} /\left(p_{1}+p_{2}+\ldots+p_{n}\right) * \Delta x \\
& y_{n}^{\prime}=y_{n}+p_{n} /\left(p_{1}+p_{2}+\ldots+p_{n}\right) * \Delta y
\end{aligned}
$$

Where, $\mathrm{n}$ is the number of all points belonging to the line to be connected. When the value of $\mathrm{n}$ increases, the value of $p_{n} /\left(p_{1}+p_{2}+\ldots+p_{n}\right)$ will decrease, which can reach the purpose of attenuation.

The effect of this method can be seen in the following Fig. 6. Fig. 6a shows that a gap between two lines in different scale charts. When the attenuation edge-matching method based 
on error distribution theory is used to connect the gap, the result is shown in Fig. 6b. As is shown, the lines can be matched smoothly and can maintain the trend as they are.

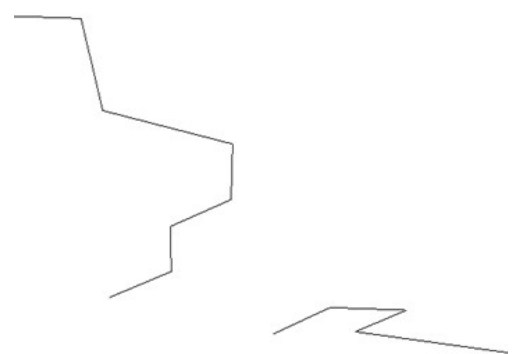

(a) A Gap between Two Lines in Different Scale Charts

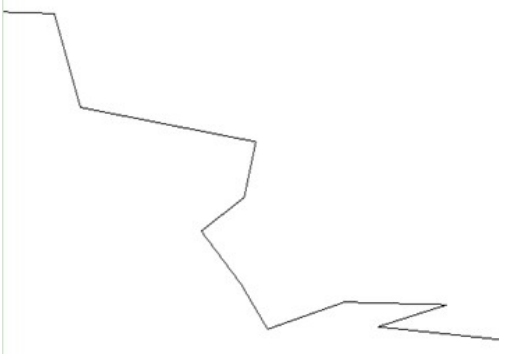

(b) Splicing Result of the Gap

Figure 6 :Effect of the Attenuation Edge-matching Method

\section{Numerical Example}

In order to verify the effectiveness of this algorithm, 13 pieces of S-57 standard electronic charts around Rizhao Port are taken as an example. Firstly, an appropriate data extraction program is written to extract the radar data, then the forward radius $\mathrm{R}$ and the maximum number $\mathrm{M}$ are set respectively according to the discrete point of the boundary line in different charts. As is shown in Table 1, the points which are not satisfied with the algorithm are deleted. For example, the original radar points of chart $\mathrm{C} 1312300$ are 1967, the forward radius R is set as 3000 and the maximum number $\mathrm{M}$ is set as 5 by observing Fig. 1 and Fig. 2. After clipping, we can get 1925 points of chart $\mathrm{C} 1312300$ and we can get 26116 points of 13 charts before merging in Rizhao port. Then the buffers are built to merge and de-duplicate data according to the characteristics of the data fragmentation; as a result, the data with low accuracy are deleted. Finally, the gaps between charts are connected and the remaining points are 10669.

The radar simulation data connected are shown in Fig. 7. Contrasting the radar images around Rizhao Port before and after splice, we can see that all boundary lines are cut off, all redundant data are deleted and all gaps are connected. It is obviously that the radar simulation image displays the real coastline accurately.

\begin{tabular}{|c|c|c|c|c|c|c|}
\hline Chart name & $\begin{array}{l}\text { Original radar } \\
\text { points number }\end{array}$ & $\begin{array}{l}\text { Forward } \\
\text { radius }\end{array}$ & $\begin{array}{l}\text { The maximum } \\
\text { number }\end{array}$ & $\begin{array}{c}\text { Points } \\
\text { number } \\
\text { after clipping }\end{array}$ & $\begin{array}{l}\text { Points number } \\
\text { before } \\
\text { merging }\end{array}$ & $\begin{array}{l}\text { Points number } \\
\text { after splicing }\end{array}$ \\
\hline C1312300 & 1967 & 3000 & 5 & 1925 & \multirow{13}{*}{26116} & \multirow{13}{*}{10669} \\
\hline $\mathrm{C} 1312500$ & 2428 & 6000 & 5 & 2373 & & \\
\hline CN336002 & 4301 & 3000 & 5 & 4259 & & \\
\hline $\mathrm{C} 1412310$ & 4323 & 4000 & 5 & 4291 & & \\
\hline C1412510 & 849 & 3500 & 5 & 838 & & \\
\hline $\mathrm{C} 1412570$ & 2358 & 8000 & 5 & 2337 & & \\
\hline C1512571 & 515 & 4000 & 5 & 509 & & \\
\hline CN336001 & 3617 & 4000 & 5 & 3594 & & \\
\hline CN337001 & 3215 & 4000 & 5 & 3154 & & \\
\hline CN437101 & 274 & 3116 & 5 & 248 & & \\
\hline CN437111 & 860 & 2000 & 5 & 843 & & \\
\hline CN437122 & 949 & 2000 & 5 & 940 & & \\
\hline CN537112 & 809 & 2000 & 5 & 805 & & \\
\hline
\end{tabular}

Table 1: Changes in Radar Simulation Data 

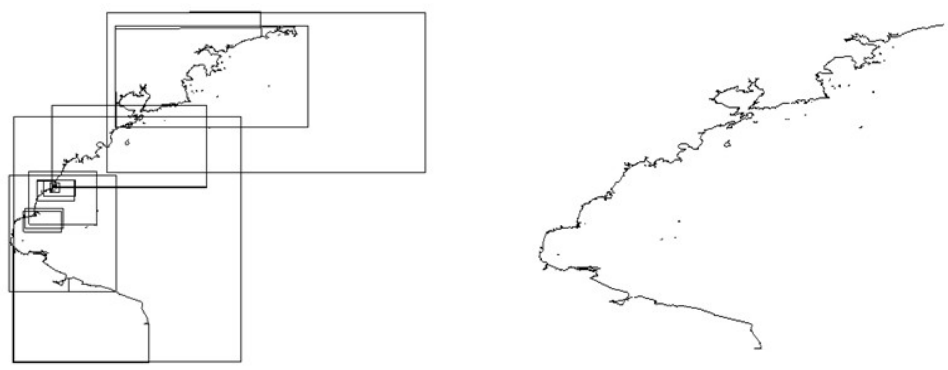

(a) The Original Radar Simulation Data

(b) The Radar Simulation Data after Splicing

Figure 7: The Radar Simulation Data around Rizhao Port

\section{Conclusion}

In this paper, different scales of radar simulation data have been seamlessly spliced by using the generation algorithm, which is suitable for processing large amount of data, multiple map sheet and data from a variety of scales. The whole process includes data preprocessing, boundary line cutting, data merging and gap connecting. The algorithm performs with high efficiency and it reduces the manual intervention in stitching. From Fig. 7, we can see that the boundary lines of radar simulation data have been cut off, the redundant data have been deleted and the gaps have been connected. In a word, the radar simulation data have been seamlessly spliced, which can meet the practical need of navigation simulator; however, there are also some shortcomings in the generation algorithm. When the points in coastline are more decentralized than that in boundary line, they will be deleted accidentally. In the next publication, we will discuss how to solve this problem and generate a more accurate image. The generation algorithm proposed in the paper can be applied to more fields, like splicing of contour lines, generation of three-dimensional visual terrain and cartography.

\section{References}

[1] S. Yao, X.Y. Zhang, Y. Yin, X. Xiong, J. Lin. The Merging Algorithm of Radar Simulation Data in Navigation Simulator[C].14th International Conference on Algorithm and Architectures for Parallel Processing. Springer Verlag, Heidelberg. pp. 516-524(2014).

[2] C. G. Sui, R. C. Peng. The Elementary Introduction to the Seamless Contiguity of Digital Chart [J]. Hydrographic Surveying and Charting. 23(5), 36-39(2003) (In Chinese).

[3] G. S. Han, B. G. Yang, J. L. Guo, X. Y. Luo, J. J. Yuan. Key Technology and Software Implementation for Physical Seamless Contiguity of Crossing Feature [J]. Science of Surveying and Mapping, 35(1), 119-120(2010) (In Chinese).

[4] Y. Liu, Y. Liu, Z. Yang. The Design and Implementation of Large-scale Topographic Map Data Splicing Program [J]. Urban Geotechnical Investigation \& Surveying. (1), 65-67(2012) (In Chinese).

[5] H. P. Huang, Y. P. Han, J. F. Zhang. Design and of Edge Matching Algorithm of Intersected Contour Lines [J]. Journal of North China University of Water Resource and Electric Power (Nature Science Edition). 35(3), 62-65(2014) (In Chinese).

[6] Y. Zhang, R. F Wang, X. J. Liao. Algorithm of Virtual Stitching on Digital Maps Edge Matching [J]. Computer Engineer and Design. 31(16), 3640-3643(2010) (In Chinese).

[7] J. Pouderoux, S. Spinello. Global Contour Lines Reconstruction in Topographic Maps[C]. Ninth International Conference on Document Analysis and Recognition (Volume: 2). pp.779-783(2007). 
[8] B. Sandhya, A. Agarwal, C. Raghavendra Rao, R. Wankar. Automatic Gap Identification towards Efficient Contour Line Reconstruction in Topographic Maps[C]. Third Asia International Conference on Modelling \& Simulation. pp. 309-314(2009).

[9] C. G. Sui, R. S. Fang, L. W. Yin. Seamless Contiguity of Digital Chart [J]. Geomatics and Information Science of Wuhan University. 30(3), 278-281(2005) (In Chinese).

[10] D. N. Veritas. Standard for Certification No.2.14 Maritime Simulator Systems [M]. Norske Veritas, Oslo. pp.22 (2011).

[11] J. H. Zhao. Arithmetic Design and the Realization if the Function of Trend-edge matching [J]. Bulletin of Surveying and Mapping.50(6), 26-27(2004) (In Chinese). 